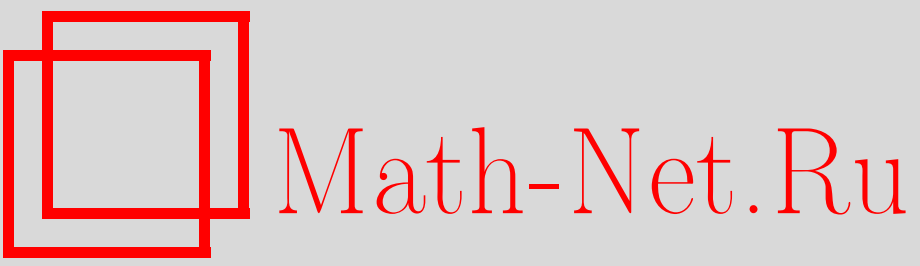

В. В. Яблоков, Об усреднении решений задачи Неймана для системы Ламе теории упругости в области, часть которой представляет собой совокупность тонких цилиндров, УМН, 2003, том 58, выпуск 4, 167-168

DOI: https://doi.org/10.4213/rm658

Использование Общероссийского математического портала Math-Net.Ru подразумевает, что вы прочитали и согласны с пользовательским соглашением

http://www.mathnet.ru/rus/agreement

Параметры загрузки:

IP : 54.147 .182 .235

26 апреля 2023 г., 15:08:12 


\title{
ОБ УСРЕДНЕНИИ РЕШЕНИЙ ЗАДАЧИ НЕЙМАНА ДЛЯ СИСТЕМЫ ЛАМЕ ТЕОРИИ УПРУГОСТИ В ОБЛАСТИ, ЧАСТЬ КОТОРОЙ ПРЕДСТАВЛЯЕТ СОБОЙ СОВОКУПНОСТЬ ТОНКИХ цИЛИНДРОВ
}

\author{
В. В. Яьлоков
}

В работе рассматривается задача усреднения решений системы Ламе линейной теории упругости в областях с тонкими каналами, представляющими собой прямые цилиндры в $\mathbb{R}^{2}$ длины $\varepsilon^{q}(\varepsilon-$ малый параметр, $q=$ const $>0)$ и радиуса $a_{\varepsilon}=O\left(\varepsilon^{1+q}\right)$, количество каналов $N_{\varepsilon}=O\left(\varepsilon^{-1}\right)$. На границе области ставится краевое условие Неймана. Такого рода задачи с использованием более сложной техники впервые изучались в [1]. Для оператора Лапласа задача рассмотрена в [2]. Автор исследует асимптотику решения при $\varepsilon \rightarrow 0$, выписывает предельную задачу и получает оценку близости решений исходной и пределшной задач.

Рассмотрим ограниченную область $\Omega$ в $\mathbb{R}^{2}$ с гладкой границей. Положим $\Omega^{+}=\Omega \cap\left\{x_{1}>0\right\}$, $\Omega^{-}=\Omega \cap\left\{x_{1}<0\right\}, \gamma=\Omega \cap\left\{x_{1}=0\right\} \neq \varnothing$. Определим цилиндр $T_{\varepsilon}^{0}=\left\{0<x_{1}<\varepsilon^{q}\right.$, $q=$ const $\left.>0,\left|x_{2}\right|<a_{\varepsilon}\right\}$. Пусть $T_{\varepsilon}=\bigcup_{j=1}^{N_{\varepsilon}} T_{\varepsilon}^{j}$ - объединение цилиндров вида $T_{\varepsilon}^{0}+\varepsilon z$, лежащих целиком внутри области $\Omega$, где $z \in \mathbb{Z}_{1}$ - множество векторов вида $\left(0, z_{2}\right)$ с целочисленньми координатами. Положим $\Omega_{\varepsilon}^{+}=\Omega^{+} \cap\left\{x_{1}>\varepsilon^{q}\right\}, \gamma_{\varepsilon}=\Omega^{+} \cap\left\{x_{1}=\varepsilon^{q}\right\}, G_{\varepsilon}^{0}=\gamma \cap \partial T_{\varepsilon}$, $\gamma_{\varepsilon}^{0}=\gamma \backslash G_{\varepsilon}^{0}, G_{\varepsilon}^{1}=\gamma_{\varepsilon} \cap \partial T_{\varepsilon}, \gamma_{\varepsilon}^{1}=\gamma_{\varepsilon} \backslash G_{\varepsilon}^{1}$.

Пусть $f(x)=\left(f_{1}(x), f_{2}(x)\right)^{t}, f_{i} \in L_{2}(\Omega), i=1,2$. Символ " $t$ " означает транспонирование.

Рассмотрим в области $\Omega_{\varepsilon}$ оператор стационарной системы линейной теории упругости $\Lambda[u]=\sum_{m, k=1}^{2} \frac{\partial}{\partial x_{m}}\left(A^{m k} \frac{\partial u}{\partial x_{k}}\right)$. Здесь $u=\left(u_{1}(x), u_{2}(x)\right)^{t}$ - вектор-столбец с компонентами $u_{1}(x), u_{2}(x)$; функции $u_{i}$ определены в области $\Omega_{\varepsilon} ; A^{m k}$ - матрицы $2 \times 2$ с компонентами $a_{i j}^{m k}=\lambda \delta_{i m} \delta_{j k}+\mu \delta_{i j} \delta_{m k}+\mu \delta_{i k} \delta_{m j}$, где $\lambda=$ const $>0, \mu=$ const $>0$ - постоянные Ламе; $\delta_{i j}$ - символ Кронекера. Далее мы будем опускать знак суммы, подразумевая суммирование по повторяющимся индексам.

Предположим, что $\lim _{\varepsilon \rightarrow 0} a_{\varepsilon} \varepsilon^{-1-q}=\beta=$ const $>0$.

В области $\Omega_{\varepsilon}=\Omega^{-} \cup \Omega_{\varepsilon}^{+} \cup T_{\varepsilon} \cup G_{\varepsilon}^{0} \cup G_{\varepsilon}^{1}$ рассмотрим задачу

$$
\left\{\begin{array}{l}
\Lambda\left[u_{\varepsilon}\right] \equiv-\frac{\partial}{\partial x_{m}}\left(A^{m k} \frac{\partial u_{\varepsilon}}{\partial x_{k}}\right)+u_{\varepsilon}=f(x) \text { в } \Omega_{\varepsilon} \\
\sigma\left(u_{\varepsilon}\right) \equiv \nu_{m} A^{m k} \frac{\partial u_{\varepsilon}}{\partial x_{k}}=0 \text { на } \partial \Omega_{\varepsilon}
\end{array}\right.
$$

где $\nu=\left(\nu_{1}, \nu_{2}\right)$ - вектор внешней единичной нормали к $\Omega_{\varepsilon}$. Предположим, что $u_{\varepsilon} \in H_{1}\left(\Omega_{\varepsilon}\right)$.

С помощью леммы Лакса-Милграма легко доказать существование и единственность решения задачи (1). Кроме того, справедлива оценка $\left\|u_{\varepsilon}\right\|_{H_{1}\left(\Omega_{\varepsilon}^{+}\right)}^{2}+\left\|u_{\varepsilon}\right\|_{H_{1}\left(\Omega^{-}\right)}^{2}+\left\|u_{\varepsilon}\right\|_{L_{2}\left(T_{\varepsilon}\right)}^{2} \leqslant K_{1}$. Здесь и далее все $K_{i}=$ const $>0$ не зависят от $\varepsilon$.

Имеет место лемма, доказанная в [2].

ЛЕмма 1. Предположим, что $\lim _{\varepsilon \rightarrow 0} a_{\varepsilon} \varepsilon^{-1-q}=\beta=$ const $>0$. Тогда для функции $g(x) \in H_{1}\left(\Omega^{-}\right)$имеет место оценка:

$$
\left|\int_{\gamma} g(x) d x_{2}-\beta^{-1} \varepsilon^{-q} \int_{G_{\varepsilon}^{0}} g(x) d x_{2}\right| \leqslant K_{2}\left(\left|\beta-a_{\varepsilon} \varepsilon^{-1-q}\right|+\sqrt{\varepsilon}+\varepsilon^{q / 2}\right)\|\nabla g\|_{H_{1}\left(\Omega^{-}\right)} .
$$


Пусть $v(x)=\left(v_{1}(x), v_{2}(x)\right)-$ решение задачи

$$
\left\{\begin{array}{l}
\Lambda[v]=f \text { в } \Omega^{+} \cup \Omega^{-} \\
(\lambda+2 \mu)\left(\frac{\partial v}{\partial x_{1}}\right)_{1}+\lambda\left(\frac{\partial v}{\partial x_{2}}\right)_{2}=\beta \frac{(\lambda+2 \mu)^{2}-\lambda^{2}}{\lambda+2 \mu}[v]_{1} \text { на } \gamma \\
\left(\frac{\partial v}{\partial x_{1}}\right)_{2}+\left(\frac{\partial v}{\partial x_{2}}\right)_{1}=0 \text { на } \gamma
\end{array}\right.
$$

где $[h]=h\left(+0, x_{2}\right)-h\left(-0, x_{2}\right)-$ скачок функции, $(h)_{i}-i$-я компонента вектора, $i=1,2$.

Под решением задачи (2) будем понимать вектор-функцию $v \in H_{1}\left(\Omega^{+}\right), v \in H_{1}\left(\Omega^{-}\right)$, удовлетворяющую интегральному тождеству

$$
\begin{aligned}
& \int_{\Omega^{+}} A^{m k} \frac{\partial v}{\partial x_{k}} \frac{\partial \varphi}{\partial x_{m}} d x+\int_{\Omega^{-}} A^{m k} \frac{\partial v}{\partial x_{k}} \frac{\partial \varphi}{\partial x_{m}} d x \\
& +\int_{\Omega} v \varphi d x+\beta \frac{(\lambda+2 \mu)^{2}-\lambda^{2}}{\lambda+2 \mu} \int_{\gamma}[v]_{1}[\varphi]_{1} d x=\int_{\Omega} f \varphi d x
\end{aligned}
$$

для произвольной функции $\varphi \in H_{1}\left(\Omega^{+}\right), \varphi \in H_{1}\left(\Omega^{-}\right)$.

Существование и единственность обобщенного решения задачи (2) доказывается с помощью леммы Лакса-Мильграма. Будем предполагать, что $v \in C^{2}\left(\bar{\Omega}^{-}\right), v \in C^{2}\left(\bar{\Omega}^{+}\right)$.

Из интегральных тождеств для задач (1) и $(2)$, используя лемму 1 , гладкость функции $v$ и неравенство Корна, можно получить оценку

$\int_{\Omega_{\varepsilon}}\left(u_{\varepsilon}-v\right) d x+\int_{\Omega_{\varepsilon}^{+}}\left|\nabla\left(u_{\varepsilon}-v\right)\right|^{2} d x+\int_{\Omega^{-}}\left|\nabla\left(u_{\varepsilon}-v\right)\right|^{2} d x \leqslant K_{3}\left(\varepsilon^{q / 2}+\sqrt{\varepsilon}+\left|\beta-a_{\varepsilon} \varepsilon^{-1-q}\right|\right)$.

ТЕОРема. Пусть функчия $u_{\varepsilon}$ - обобщенное решение задачи (1), а $v \in C^{2}\left(\bar{\Omega}^{-}\right), v \in$ $C^{2}\left(\bar{\Omega}^{+}\right)$- решение задачи (2). Пусть $\lim _{\varepsilon \rightarrow 0} a_{\varepsilon} \varepsilon^{-1-q}=\beta=$ const $>0$, где $q=$ const $>0$. Тогда имеет место оченка (3).

Заметим, что если отказаться от гладкости функции $v$, то аналогичным методом можно доказать слабую в $H_{1}$ сходимость $u_{\varepsilon} \mathrm{K} v$.

В заключение автор выражает благодарность проф. Т. А. Шапошниковой за постановку задачи и постоянньй интерес к работе.

\section{СПИСОК ЛИТЕРАТУРЫ}

[1] В. А. Марченко, Е. Я. Хруслов. Краевые задачи в областях с мелкозернистой границей. Киев: Наукова думка, 1974. [2] Т. А. Шапошникова // Дифференц. уравнения. 2001. T. 37. №9. С. $1250-1257$.

Московский государственньй

Принято редколлегией университет им. М. В. Ломоносова 14.07 .2003 